\section{Absorption of Heavy Cosmic Ray Particles}

IN a previous note ${ }^{1}$ we have described experiments with the object of obtaining information about the radiation producing the heavy particles in the cosmic rays. A set of Ilford half-tone plates (70 $\mu$ thick), sensitive to $\alpha$-particles and protons and covered with thicknesses of lead up to $12 \cdot 2 \mathrm{~cm}$, have been exposed to cosmic rays on the Jungfraujoch and at Bristol for a period of 230 days. It was found that the primary radiation producing the tracks is strongly absorbed in air, but very little absorbed in lead, and it was concluded that the majority of tracks observed in the emulsion are produced by the neutron component of the cosmic rays.

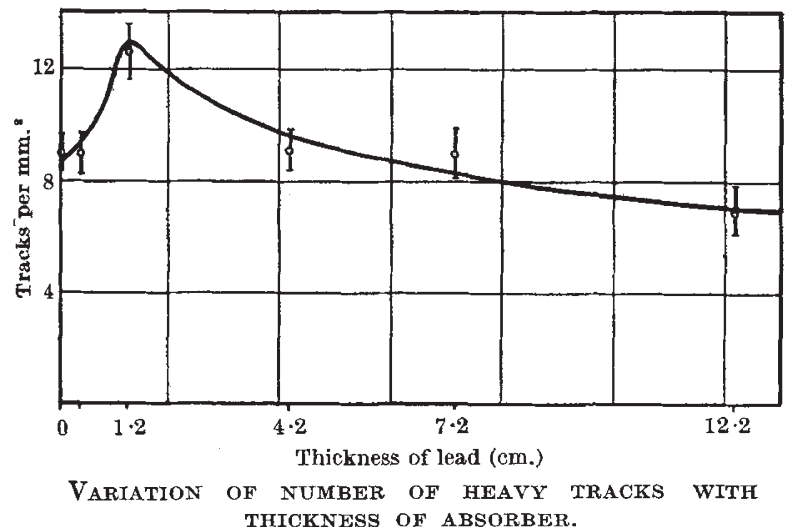

We have now measured the absorption curve in lead, on the Jungfraujoch material, in more detail and with greater accuracy. The result is shown in Fig. 1, where we have plotted the number of tracks per $\mathrm{mm} .^{2}$ against the thickness of lead. Only tracks with a visible range greater than $10 \mathrm{~cm}$. air equivalent have been included since a large fraction of the shorter tracks are due to radioactive contamination. The curve shows a maximum at about $1.2 \mathrm{~cm}$. lead.

It follows from this result that there are at least two components of cosmic radiation producing heavy tracks. The first component is very little absorbed in lead and consists very probably of neutrons. The second component has a transition curve in lead very similar to that of the soft radiation. It has a maximum coinciding precisely with the Rossi maximum and falls rapidly to a small value within $5 \mathrm{~cm}$. of lead. We therefore conclude that the soft radiation (electrons and light quanta) is responsible for part of the heavy tracks and particularly for the maximum of our curve. It is plausible to assume that the process by which these tracks are produced is the direct nuclear photo-electric effect.

The cross-section for this photo-electric effect can only be estimated very roughly from these results for several reasons. First, the number of light quanta per $\mathrm{cm} .{ }^{2} \mathrm{~min}$. at the Jungfraujoch is not known accurately. Secondly, since most of our tracks are passing through the whole emulsion, we do not know the actual distribution of range of our particles and, consequently, we do not know whether the bulk of our tracks originate from the lead or from the glass.

The number of soft ionizing particles at the Jungfraujoch is about 2 per $\mathrm{cm} .{ }^{2}$ min. After cascade multiplication in $1.2 \mathrm{~cm}$. lead, we can assume that the number of light quanta with energies greater than $10 \mathrm{Mev}$. is about 6 per $\mathrm{cm} .^{2} \mathrm{~min}$. Assuming, further, an average range of the disintegration protons of $1 \mathrm{~m}$. air equivalent, we find an average cross-section for photo-disintegration with proton emission of $10^{-25} \mathrm{~cm} .^{2}$ per lead atom or $\mathrm{SiO}_{2}$ molecule. This figure is very rough and may well be wrong by a factor 5 . We may compare it with the cross-section for the photo-electric effect with neutron emission obtained by Bothe and Gentner ${ }^{2}$ for copper and the lithium $\gamma$-rays, namely, $5 \times 10^{-28} \mathrm{~cm} .^{2}$. Our figure may seem to be rather large, especially in view of the fact that neutron emission is much more probable than proton emission, but taking into account the many uncertainties involved, it is probably a reasonable order of magnitude.

If it is true that some of the protons are produced by photo-electric effect, it must be expected that an even larger number of neutrons is produced in the same way. Since we do not know the cross-sections for the photo-electric effect for oxygen and nitrogen atoms, it is difficult to decide whether the whole neutron component of cosmic radiation can be accounted for by a photo-electric effect taking place in the high atmosphere, or whether other processes are involved in the production of the neutron component too (compare also ref. 3 ).

Further details will be published elsewhere.

\section{H. Wills Physical Laboratory, C. F Power. University of Bristol. H. Hetruer.} June 3.

${ }^{1}$ Heitler, Powell and Fertel, NATORE, 144, 283 (1939).

2 Bothe and Gentner, Z. Phys., 106, 236 (1937).

${ }^{3}$ Bethe, Korff and Placzek, Phys. Rev., 57, 573 (1940).

\section{Specific Ionization of Cosmic Ray Particles}

MEASUREMENTS of the specific ionization of cosmic ray electrons with energies between $0.1 \times 10^{6} \mathrm{ev}$. and $30 \times 10^{6} \mathrm{ev}$. have been made by Corson and Brode ${ }^{1}$ by counting droplets on photographs taken with a counter-controlled cloud chamber using a delayed expansion. They established the existence of a minimum ionization at about $1.5 \times 10^{6} \mathrm{ev}$. and a logarithmic increase in ionization with energy beyond this minimum. Using essentially the same method, I have measured the specific ionization of cosmic ray particles over the energy range $2 \times 10^{8}-6 \times 10^{9} \mathrm{ev}$.

The track photographs were classified in the following way :

(1) In general, all particles in showers were assumed to be electrons. The exceptions were a few particles of energy greater than $10^{8} \mathrm{ev}$. which showed an ionization markedly less than that of electrons of the same energy, and were therefore considered to be mesons responsible for the production of 'knock on' showers. Three particles, each from a separate shower, were taken as mesons out of forty measured showers for this reason.

(2) Single particles with energy less than $40 \times$ $10^{6}$ ev. not exhibiting heavy ionization were taken as electrons. Mesons of this energy would be recognizable because of their heavy ionization.

(3) Single particles in the energy region $2 \times 10^{8} \mathrm{ev}$. to $7 \times 10^{8} \mathrm{ev}$. showing markedly heavy ionization were taken as protons.

(4) All other single particles were assumed to be mesons, althoughcertainly a small fraction were protons.

The theoretical curves for electrons, mesons $(M=170 \mathrm{~m})$ and protons are shown in the accom panying graph. The experimental results for electrons show good agreement with the theory over the 